\title{
O Estado da Arte sobre a Teoria da Contingência: um Estudo Bibliométrico entre o Período de 2015 a 2019
}

The State of the Art on Contingency Theory: A Bibliometric Study Between the Period 2015 to 2019

EI Estado del Arte sobre la Teoría de Contingencia: un Estudio Bibliometrico entre el Periodo 2015 a 2019

Maico Schnell ${ }^{1}$ Mestre em Contabilidade ${ }^{2}$ maicoschnell@gmail.com http://lattes.cnpq.br/1945681756741891

Pedro Junior de Oliveira Trocz

Mestrando em Contabilidade 2 pedrojunior94@yahoo.com http://lattes.cnpq.br/9328031751773659

Universidade do Norte do Paraná, UNOPAR, Brasil ${ }^{1}$ Universidade Estadual do Oeste do Paraná, UNIOESTE, Brasil ${ }^{2}$

Artigo recebido em 25.04.2020. Última versão recebida em 04.06.2020. Aprovado em 08.06.2020. 


\title{
Resumo
}

O objetivo deste trabalho é mapear as publicações científicas sobre a Teoria da Contingência. Para isso foi realizada uma análise bibliométrica das pesquisas publicadas na Web of Science, entre 2015 e 2019. Os dados foram importados para o software HistCite ${ }^{\circledR}$ para organização dos artigos em referências mais citados, países que mais publicaram, fonte das publicações dos artigos publicados e com maiores impactos. Para isso esses dados foram criadas as tabelas e figuras para representar os indicadores bibliométricos. Os resultados apontaram que foram localizados 326 artigos sendo escritos por 840 pesquisadores com vínculo em 452 instituições, ainda foi possível identificar que os artigos foram publicados em 64 países estando presente em 210 periódicos e os mesmos utilizaram 19.113 referências, bem como 1.072 palavras-chave. Este estudo também permitiu identificar as áreas os 10 mais recentes no ano de 2019. Como conclusões os resultados encontrados retratam o interesse dos pesquisadores em estudar a Teoria da Contingência nos últimos 5 anos em âmbito internacional.

Palavras-chave: Teoria da Contingência. Organizações. Bibliometria.

\begin{abstract}
The objective of this work is to map the scientific publications on contingency theory. For this, a bibliometric analysis of the research published on the Web of Science between 2015 and 2019 was performed. The data were imported into the HistCite software ${ }^{\circledR}$ to organize the articles in the most cited references, countries that published the most, source of publications of published articles and with greater impacts. For this, these data were created the tables and figures to represent the bibliometric indicators. The results showed that 326 articles were found being written by 840 researchers with links in 452 institutions, it was still possible to identify that the articles were published in 64 countries being present in 210 journals and they used 19,113 references, as well as 1,072 keywords. This study also identified the areas the 10 most recent in 2019. As conclusions the results found portray the interest of researchers in studying contingency theory in the last 5 years internationally.
\end{abstract}

Keywords: Contingency Theory. Organizations. Bibliometry.

\section{Resumen}

El objetivo de este trabajo es mapear las publicaciones científicas sobre la teoría de contingencias. Para ello, se realizó un análisis bibliométrico de la investigación publicada en la Web of Science entre 2015 y 2019. Los datos fueron importados en el software HistCite ${ }^{\circledR}$ para organizar los artículos en las referencias más citadas, los países que más publicaron, fuente de publicaciones de artículos publicados y con mayores impactos. Para ello, estos datos se crearon las tablas y figuras para representar los indicadores bibliométricos. Los resultados mostraron que se encontraron 326 artículos escritos por 840 investigadores con enlaces en 452 instituciones, todavía era posible identificar que los artículos fueron publicados en 64 países presentes en 210 revistas y utilizaron 19.113 referencias, así como 1.072 palabras clave. Este estudio también identificó las áreas más recientes en 2019. Como conclusiones, los resultados encontrados retratan el interés de los investigadores en estudiar la teoría de contingencias en los últimos 5 años a nivel internacional.

Palabras clave: Teoría de contingencias. Organizaciones. Bibliometría. 
O Estado da Arte sobre a Teoria da Contingência: um Estudo Bibliométrico entre o Período de 2015 a 2019

\section{INTRODUÇÃO}

Os sistemas de controle contingências são cada vez mais necessários para operar em limites organizacionais. Para tanto, a ideia de contingência requer mais esclarecimentos, pois se pode argumentar que todas as pesquisas sobre esses temas têm de tomar uma abordagem de "contingência", pois torna-se reconhecido que as soluções universais para problemas no controle organizacional geralmente não existem (Otley, 2016). Com isso, ao passar do tempo, vários foram os métodos desenvolvidos para gerenciar, controlar, planejar e organizar as empresas, sempre na busca do melhor modelo de administrar (Fagundes et al. 2011).

Dentre as perspectivas da Teoria da Contingência, vários são os estudos seminais em destaque: Woodward (1958, 1965); Burns e Stalker (1960; Chandler (1962); Lawrence e Lorsch (1967), Perrow (1976). E ainda estudos mais recentes das últimas 2 décadas: Chenhall (2003), Tillema (2005), Aguiar e Frezatti (2007) e Cadez (2007) Chen (2008), os quais estudaram a teoria nos mais variados campos da pesquisa, desde questões orçamentárias; tecnologia como fator de impacto; o ambiente externo mecânico e orgânico; bem como estratégia e estrutura das organizações. Porém, quais são os pesquisadores contemporâneos dos últimos 5 anos?

Assim, para preencher essa lacuna de pesquisa, faz-se pertinente apresentar os pesquisadores internacionais e mais recentes sobre a Teoria da Contingência. Baseado neste contexto, a pesquisa bibliométrica ganha importância ao retratar este cenário.

Diante das premissas mencionadas no qual destaca publicações sobre a Teoria da Contingência, o estudo concentra-se na resposta do seguinte questionamento: Como se apresenta a produção científica internacional sobre a Teoria da Contigência na Web of Science entre 2015 a 2019? E como forma de responder a essa questão, o estudo tem como objetivo identificar os artigos publicados com maior grau de impacto, países mais produtivos, os autores mais citados, as áreas de pesquisa e publicações, as referências mais utilizadas entre outros, por meio de uma análise bibliométrica.

Este artigo está estruturado em cinco partes como: após esta introdução, inicia-se o referencial teórico, na sequência os procedimentos metodológicos, análise e discussão dos resultados apresentados, considerações finais e por fim, seguida da lista de referências das obras citadas neste trabalho.

\section{REVISÃO DE LITERATURA}

\subsection{Teoria da Contingência}

A teoria da contingência tem como objetivo explicar sobre os fatores organizacionais que podem vir a interferir no processo de tomada de decisão das empresas, levando em consideração ambientes incertos e o nível de informações disponíveis (Soares, Cavalcante, \& Santos, 2019). Sua origem foi no final da década de 1950 e início da década de 1960, consolidando elevada amplitude de estudo das questões que foram iniciadas pela Escola Sistêmica (Oliveira, 2008).Pois de acordo com Storner e Freeman (1985), a organização é vista como um sistema composto de subsistemas (departamentos, unidades) que se relacionam e interagem para sua sobrevivência. Interações que ocorrem tanto interna quanto externamente, interatuando com ambientes. Neste mesmo sentido (Chenhall, 2003) afirma que perante a teoria contingencial, as 
O Estado da Arte sobre a Teoria da Contingência: um Estudo Bibliométrico entre o Período de 2015 a 2019

organizações são percebidas como sistemas abertos que precisam readequar-se constantemente sua maneira gestão, a fim de manter ou melhorar seu desempenho.

Os estudos sobre a teoria da contingência começaram a desenvolver com o objetivo de expor que não existe um único modelo de estrutura organizacional para todas as organizações, contradizendo a escola clássica de administração, a qual ressaltava que uma única estrutura organizacional era eficiente e eficaz para qualquer tipo de empresa, seja ela de pequeno, seja de médio ou grande porte (Donaldson, 1999). Sendo assim, com o desenvolvimento de novos estudos com o passar do tempo, se percebe que existe muitas formas de se administrar e não uma melhor maneira, visto que uma organização precisa estar sempre se adaptando às mudanças ambientais (Camacho, 2010). Corroborando com está ideia Junqueira (2010) expõe, que cada empresa necessite buscar a sua melhor maneira de se estruturar com o intuito de atingir suas metas e seus objetivos.

Alguns estudos considerados precursores da teoria da contingência foram observando e categorizando contingências percebidas no ambiente organizacional, influenciando assim o contexto do sistema organizacional (Soares, Cavalcante, \& Santos, 2019). Algumas pesquisas relacionadas estão descritas a seguir no quadro 1.

\section{Quadro 1 - Relato histórico das principais pesquisas e contribuições sobre a Teoria da contingência}

\begin{tabular}{|l|l|l|}
\hline Ano & Autores & Contribuições \\
\hline 2003 & Chenhall & $\begin{array}{l}\text { Revisão sobre estudos de 1980, ressaltando a falta de estudos } \\
\text { qualitativos sobre o assunto. }\end{array}$ \\
\hline $1972-1989$ & $\begin{array}{l}\text { Wells, Egelhof, Ghoshal e } \\
\text { Norhia }\end{array}$ & $\begin{array}{l}\text { Indicam a internacionalização ou a expansão (geográfica ou } \\
\text { de produtos) como fatores contingências. }\end{array}$ \\
\hline 1969 & Hage e Aiken & $\begin{array}{l}\text { Replicaram o estudo das contingenciais empresas de saúde e } \\
\text { previdência social. }\end{array}$ \\
\hline 1968 & Pugh et al (UK & $\begin{array}{l}\text { Concluíram que o tamanho das organizações era o principal } \\
\text { fator contingencial e que quanto maior independência maior. }\end{array}$ \\
\hline 1967 & Perrow (USA) & $\begin{array}{l}\text { Concluiu que a tecnologia era um fator contingencial, mas } \\
\text { que seu uso intensivo teria capacidade de centralizar as } \\
\text { informações. }\end{array}$ \\
\hline 1967 & Lawrence e Lorsh (USA) & $\begin{array}{l}\text { Acrescentam outro fator, a variável ambiental. Também } \\
\text { forma eles que deram o nome à teoria da contingência. }\end{array}$ \\
\hline 1965 & Woodward (Uk) & $\begin{array}{l}\text { Indicou outro fator contingencial, a tecnologia. Estava } \\
\text { preocupada com a mensuração quantitativa da estrutura } \\
\text { (indústria manufatureira). }\end{array}$ \\
\hline 1962 & Chandler (UK) & $\begin{array}{l}\text { Realizou estudos sobre a diversificação e concluiu que a } \\
\text { estratégia é outro fator contingencial. }\end{array}$ \\
\hline 1939 & Burns e Stalker (UK) & $\begin{array}{l}\text { Indicam a incerteza e a inovação como fatores contingências } \\
\text { (estudo industrial eletrônica). }\end{array}$ \\
\hline
\end{tabular}

Fonte: Adaptado de Marques e Souza (2010, p.4)

Os estudos precursores da teoria da contingência tiveram como uma das contribuições, a identificação dos fatores internos e externos que podem afetar a estrutura organizacional e também mediram o nível de adequação estrutural das organizações. Entretanto, os fatores contingenciais abordados na literatura são variados, porém, mais comumente estão ambiente, tecnologia, estrutura, estratégia e porte organizacional (Espejo \& Frezatti, 2008). Ademais, Aguiar e Frezatti (2007), definem como fatores contingenciais as variáveis contingenciais que afetam a gestão das organizações, podendo ser classificadas como externas, aquelas que não possuem controle pela organização, e variáveis internas, controláveis totalmente ou 
O Estado da Arte sobre a Teoria da Contingência: um Estudo Bibliométrico entre o Período de 2015 a 2019

parcialmente pela empresa. Na Tabela 1, a qual foi retirada do estudo de Klein, Trocz, Toigo e Wrubel (2019) encontram-se os cinco fatores contingenciais, conceitos e descrições e variáveis possíveis de serem mensuradas.

\section{Tabela 1- Fatores contingenciais e variáveis de mensuração}

\begin{tabular}{ll}
\hline Fatores & Descrição \\
\hline & "Ambiente refere-se a atributos específicos, tais como \\
concorrência intensa de preços dos concorrentes \\
existentes ou potenciais, ou probabilidade de uma \\
mudança na disponibilidade de materiais" (Chenhall, \\
2007, p.172). "A variável ambiente abrange \\
estabilidade, previsibilidade e a rapidez de mudanças \\
(Espejo e Frezatti, 2008, p.66). \\
“As inovações tecnológicas específicas de informação \\
são utilizadas de várias maneiras em diferentes \\
organizações, representam a utilização de inovações da \\
tecnologia da informação em geral. Inclui intercâmbio \\
eletrônico de dados, gestão da cadeia de abastecimento, \\
armazenamento de dados de clientes ou de gestão de \\
relacionamento contínuo. (Hyvönen, 2007, p. 353). \\
Conceito de tecnologia segundo Chenhall (2003) a \\
forma pela qual a organização transforma entradas em \\
saídas, e que incluem: hardware, pessoas, processos, \\
softwares e conhecimento.
\end{tabular}

"Estrutura organizacional é a especificação

formal de diferentes papeis para os membros da organização, ou tarefas para os grupos, para garantir que as atividades da organização sejam realizadas. "Arranjos estruturais influenciam a eficiência do trabalho, a motivação das pessoas, informação, fluxos e sistemas de controle podem ajudar a moldar o futuro da organização". (Chenhall, 2007, p. 179). Refere-se principalmente ao grau de centralização existente na organização (Gordon e Narayanan, 1984).

Chenhall (2003, p. 150) salienta que “[...] as organizações podem se posicionar em determinados ambientes. Assim, a atual gama de produtos é muito incerta, estratégia de produto em uma reformulação de mercado é mais previsível pode remover a pressão do ambiente, limitar, oportunidades potenciais e, portanto, requer que a organização examine suas atitudes para a troca entre o potencial de retorno, risco aceitável e incerteza". Segundo a tipologia de Porter (1986) dividese em baixo custo e diferenciação.

De acordo com Chenhall (2007, p. 183), existem várias maneiras de estimar o tamanho da empresa incluindo lucros, volume de vendas, ativos, valorização das ações e dos trabalhadores.

Tecnologia da informação: a) Comércio Eletrônico (página na internet que viabiliza comércio eletrônico dos seus produtos e serviços); b) CRM (gerenciamento da relação com o cliente); c) Gerenciamento da cadeia de suprimentos; d) Armazenamento de dados; e) Troca de dados de forma eletrônica; f) Softwares integrados entre áreas funcionais da empresa; g) TQM (gerenciamento da qualidade total). Tecnologia da produção: a) Grau de repetitividade das atividades de linha de produção; b) Quantas tarefas da linha de produção são as mesmas, dia após dia; c) Número de pessoas que fazem tarefas diversificadas à maioria do tempo na linha de produção da empresa.

a) Com relação ao desenvolvimento de novos produtos; b) Contratação e demissão de pessoal; c) Seleção de investimentos considerados relevantes para a empresa; d) Alocação orçamentária; e) Decisões de preços.

a) Ênfase na busca da alta qualidade do produto/serviço, maior que a concorrência; b) Oferecer suporte ao cliente do produto/serviço; c) Desenvolver características únicas do produto/serviço; d) Buscar uma imagem da marca com atributos superiores ao da concorrência; e) Investir em Pesquisa e Desenvolvimento; f) Buscar um preço de venda inferior ao aplicado pelo mercado.

Faturamento operacional bruto anual ou número de funcionários em tempo integral na empresa a depender do setor de investigação. 
O Estado da Arte sobre a Teoria da Contingência: um Estudo Bibliométrico entre o Período de 2015 a 2019

Fonte: Adaptado de Beurene Fiorentin, (2014, p.199) e Espejo e Frezatti, (2008, p.65-72).

Entende-se que os fatores contingenciais são endógenos e exógenos à empresa e que podem determinar influências as suas estratégias e estrutura organizacional (Camacho, 2010). E ainda o autor afirma que as contingências tecnologia, estrutura, porte e estratégia e, recentemente estudos indicam também o "desempenho", geram informações que impactam de alguma forma o resultado de uma empresa.

\subsection{Bibliometria}

A abordagem bibliometrica da pesquisa levou em consideração as principais leis bibliométricas, conforme Vanti (2002), são Lotka, Zipf e Bradford. A Lei de Lotka ou Lei do Quadro Inverso (1926) aponta para a medição da produtividade dos autores, mediante um modelo de distribuição de tamanho - frequência dos diversos autores, em um conjunto de documentos; a Lei de Bradfor ou Lei da Dispersão (1934) elenca que, mediante a medição da produtividade das revistas é possível estabelecer o núcleo e as áreas de dispersão sobre um determinado assunto em um mesmo conjunto de revistas. Já a Lei de Zipf ou Lei do Mínimo Esforço (1949), consiste em medir a frequência do aparecimento das palavras em vários textos, o que gera uma lista ordenada de termos de uma determinada disciplina ou assunto (Vanti, 2002). Na próxima seção, são detalhados a metodologia desenvolvida para o estudo bibliométrico.

\section{METODOLOGIA}

O presente estudo de caráter teórico buscou atender ao objetivo de analisar a produção científica internacional sobre a Teoria da Contingência, foi utilizada a bibliométrica, que consiste na aplicação de técnicas estatísticas utilizada para quantificar os processos de comunicação, escrita e emprego de indicadores bibliométricos para medir a produção científica (Oliveira, 2001). A bibliometria tem sido aplicada com a finalidade métrica de citações em diferente área do conhecimento (Machado, 2007).

Com base no trabalho de Mainardes et al. (2008) foi este estudo bibliométrico foi desenvolvido em duas etapas: (i) busca sistemática; (ii) análise descritiva dos artigos.

Na primeira etapa foi escolhido a base de dados da Web o corte temporal dos últimos 5 anos sendo o ano de partida foi 2015 até julho de 2019, data esta da presente pesquisa. Na segunda etapa, foram coletados os dados acerca da temática Teoria da Contingência, na base de dados Web of Science (WoS), e sua sub-base, Topics, obtendo como critérios de busca a palavra-chave "Contingency theory". Finalmente, os termos de busca, foram considerados com a abrangência de títulos, resumos e palavras-chave, conforme as leis de Lotka ou Lei do Quadro Inverso de 1926.

Na busca ocorrida em 31 de julho de 2019, foram encontrados 391 artigos, porém ao estabelecer o filtro document types para articles review usando o critério de extrair pesquisa com avaliações "entre pares" o resultado chegou a 326 artigos, os quais utilizados para a análise bibliométrica. Os dados foram importados para o softwareHistCite ${ }^{\circledR}$, devido à limitação do software escolhido. O formato escolhido para exportação do texto simples (TXT) com todos os campos disponíveis na base de dados. Ademais para organização dos artigos e referências mais citados, países que mais publicaram fonte das publicações com artigos publicados, e com maiores impactos, entre outros. Para isso esses dados foram criados as tabelas, figuras para apresentar os indicadores bibliométricos. 
O Estado da Arte sobre a Teoria da Contingência: um Estudo Bibliométrico entre o Período de 2015 a 2019

E na segunda etapa, foram aplicados critérios de buscas que levaram em consideração a análise qualitativa como as áreas de pesquisa e os mais citados em 2019, identificados por meio da técnica bibliométrica. Como principal limitação tem-se a generalização dos dados, visto que se restringem apenas artigos analisados na base de dados da pesquisa mencionada. A seguir os resultados discutidos na forma de tabelas e figuras dos dados analisados.

\section{ANÁLISE DOS RESULTADOS}

Nesta seção, são discutidos e apresentados os resultados da busca sistemática da literatura.

\subsection{Busca sistemática da literatura}

Conforme descrito na Metodologia a busca realizada na base de dados Web of Science (WoS), de artigos internacionais que discutem a Teoria da Contingência e sua recente relação com os mais variados temas, foram localizados 326 artigos, sendo escritos por 840 pesquisadores, com vínculo em 452 instituições de 64 países, publicados em 210 periódicos e, utilizaram 19.113 referências, bem como 1.072 palavras-chave, sendo que os dados estão apresentados na Tabela 1 , a seguir.

\begin{tabular}{lc}
\multicolumn{2}{c}{ Tabela 2- Levantamento Bibliométrico } \\
\cline { 2 - 2 } Critérios & Frequência \\
Publicações (artigos) & 326 \\
Autores & 840 \\
Fontes de publicação (periódicos) & 210 \\
Países & 64 \\
Instituições (vínculo dos autores) & 510 \\
Referências Citadas & 19.113 \\
Palavras-chave & 1.072 \\
\cline { 2 - 2 } Fonte: elaborado pelos autores, a partir da Web of Science, julho de 2019.
\end{tabular}

Nota-se que as publicações por ano, por meio da Figura 1, a frequência das mesmas no período que compreende cinco anos de 2015 e 2019. O artigo de 2015 com maior número de citações, foi de Josefy M, Kuban S, Ireland RD, Hitt MA, publicado na revista Academy Of Management Annals, intitulado, "All ThingsGreat and Small: OrganizationalSize, Boundaries of the Firm, and a ChangingEnvironment", onde os autores descrevem sobre o porte das pequenas, médias e grandes empresas e sobre as influencias organizacionais, limites da firma e um ambiente em mudança. Observa-se que a cada ano ocorreu aumento acentuado das publicações dobre o tema, atingindo o auge em 2017 com um número representativo de 82 publicações, aumento este acima da média dos últimos anos não sendo possível identificar o crescimento por se tratar de um estudo bibliométrico. 
O Estado da Arte sobre a Teoria da Contingência: um Estudo Bibliométrico entre o Período de 2015 a 2019

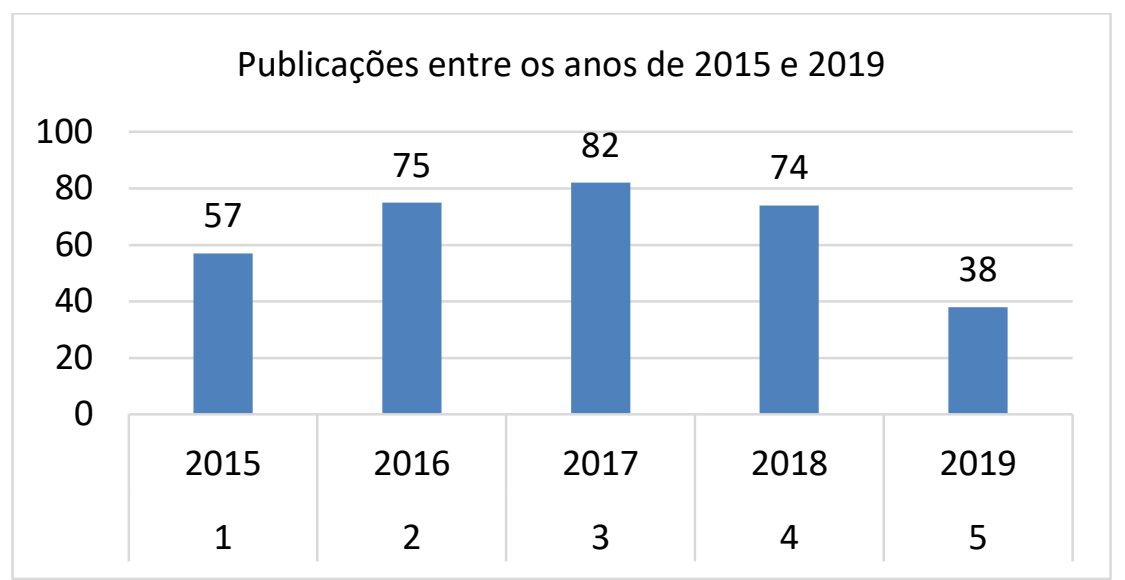

Figura 1 - publicações entre os anos de 2015 e 2019

Fonte: elaborado pelos autores, a partir da Web of Science, julho de 2019.

A partir de 2015, progressivamente a cada ano, novos artigos foram escritos, no entanto, em 2018 houve uma retração científica, com um total de 74 publicações neste ano corrente de 2019 a inserção de 38 artigos, com ressalva: apenas os sete primeiros meses de 2019, pois, os dados deste estudo foram coletados no dia 30 de julho sobre o tema Teoria da Contingência.

Na Tabela 2 é possível verificar as fontes de publicações com mais artigos publicados na área, levando em consideração o número mínimo de 4 trabalhos, bem como o grau de impacto, medido pelo indicador TGCS (Total Global Citation Score). A soma total de artigos desses periódicos é igual 67 artigos, o que corresponde a $21 \%$ da quantidade total de trabalhos analisados neste estudo. Sendo possível concluir certa pulverização entre várias revistas, ou seja, o estudo não apresentou a concentração de estudos em poucos periódicos, o que denota de outros trabalhos bibliométricos em apresentam a concentração de trabalhos em um número reduzido de revistas.

Ademais, nota-se na Tabela 2, três periódicos com a inserção de 8 trabalhos cada, os quais são: International Journal of Logistics Management; International Journal of Production Economics e International Journal of Project Management, este último, concentra o com o maior impacto (156) base no indicador TGCS.

Tabela 3 - Fontes de publicações com mais artigos publicados na área.

\begin{tabular}{lcc}
\hline \multicolumn{1}{c}{ Fonte das Publicações } & Quantidade & TGCS \\
\hline International Journal of Logistics Management & 8 & 52 \\
\hline International Journal of Production Economics & 8 & 90 \\
\hline International Journal of Project Management & 8 & 156 \\
\hline Journal of Cleaner Production & 7 & 163 \\
\hline International Journal Of Production Research & 6 & 116 \\
\hline International Journal of Operations \& Production Management & 5 & 60 \\
\hline Management Accounting Research & 5 & 90 \\
\hline European Journal Of Marketing & 4 & 11 \\
\hline Ieee Transactions On Engineering Management & 4 & 29 \\
\hline Journal Of Business Research & 4 & 3 \\
\hline Strategic Management Journal & 4 & 29 \\
\hline Supply Chain Management-An International Journal & 4 & 13 \\
\hline
\end{tabular}


O Estado da Arte sobre a Teoria da Contingência: um Estudo Bibliométrico entre o Período de 2015 a 2019

Fonte: elaborado pelo autor, a partir da Web of Science, julho de 2019.

De acordo. De acordo com os resultados, observa-se na Tabela 3 que o periódico Journal of Cleaner Production possui o maior impacto com 163 citações, seguido de International Journal of Project Management, com 156 citações e International Journal of Production Research; International Journal of Production Economics; Management Accounting Research; International Journal of Operations \& Production Management; Journal of Operations Management; International Journal of Logistics Management; Technovation e Journal of Management, estão entre as pesquisas mais recentes e os que mais publicam e tem os maiores impactos base no indicador TGCS sobre a Teoria da Contingência.

Tabela 4 - Fontes de Publicação com maior Impacto

\begin{tabular}{lcc}
\hline Fontes das Publicações & Quantidade & TGCS \\
\hline Journal of Cleaner Production & 7 & 163 \\
\hline International Journal of Project Management & 8 & 156 \\
\hline International Journal of Production Research & 6 & 116 \\
\hline International Journal of Production Economics & 8 & 90 \\
\hline Management Accounting Research & 5 & 90 \\
\hline International Journal of Operations \& ProductionManagement & 5 & 60 \\
\hline Journal of Operations Management & 3 & 58 \\
\hline International Journal of Logistics Management & 8 & 52 \\
\hline Technovation & 1 & 48 \\
\hline Journal of Management & 3 & 43 \\
\hline
\end{tabular}

Fonte: elaborado pelos autores, a partir da Web of Science, julho de 2019.

Pelo o exposto, verifica-se com a análise bibliométrica por meio do software HistCite, o país como maior número de trabalhos publicados é os Estados Unidos com 95 publicações, que correspondem aproximadamente $29 \%$ dos artigos encontrados na pesquisa, seguido do Reino Unido que apresenta frequência de 49 trabalhos. O terceiro país com mais publicações sobre o tema Teoria da Contingência é a Austrália com 34 artigos e na sequência os demais países como seus indicadores: China (32), França (19), Alemanha (19), Suécia (17), Malásia (16), Brasil (14) e Holanda (11). Vale ressaltar que o total de artigos publicados pelos top-10, somam um total de 306 artigos, equivalente a 94\% das publicações analisadas neste estudo.

Observa-se ainda, que os Estados Unidos é o Reino Unido apesar de apresentarem a maior quantidade de artigos publicados, também são os países com maiores scores de citações, no indicador TGCS, sendo respectivamente 695 e 467 citações nas últimas décadas. 
O Estado da Arte sobre a Teoria da Contingência: um Estudo Bibliométrico entre o Período de 2015 a 2019

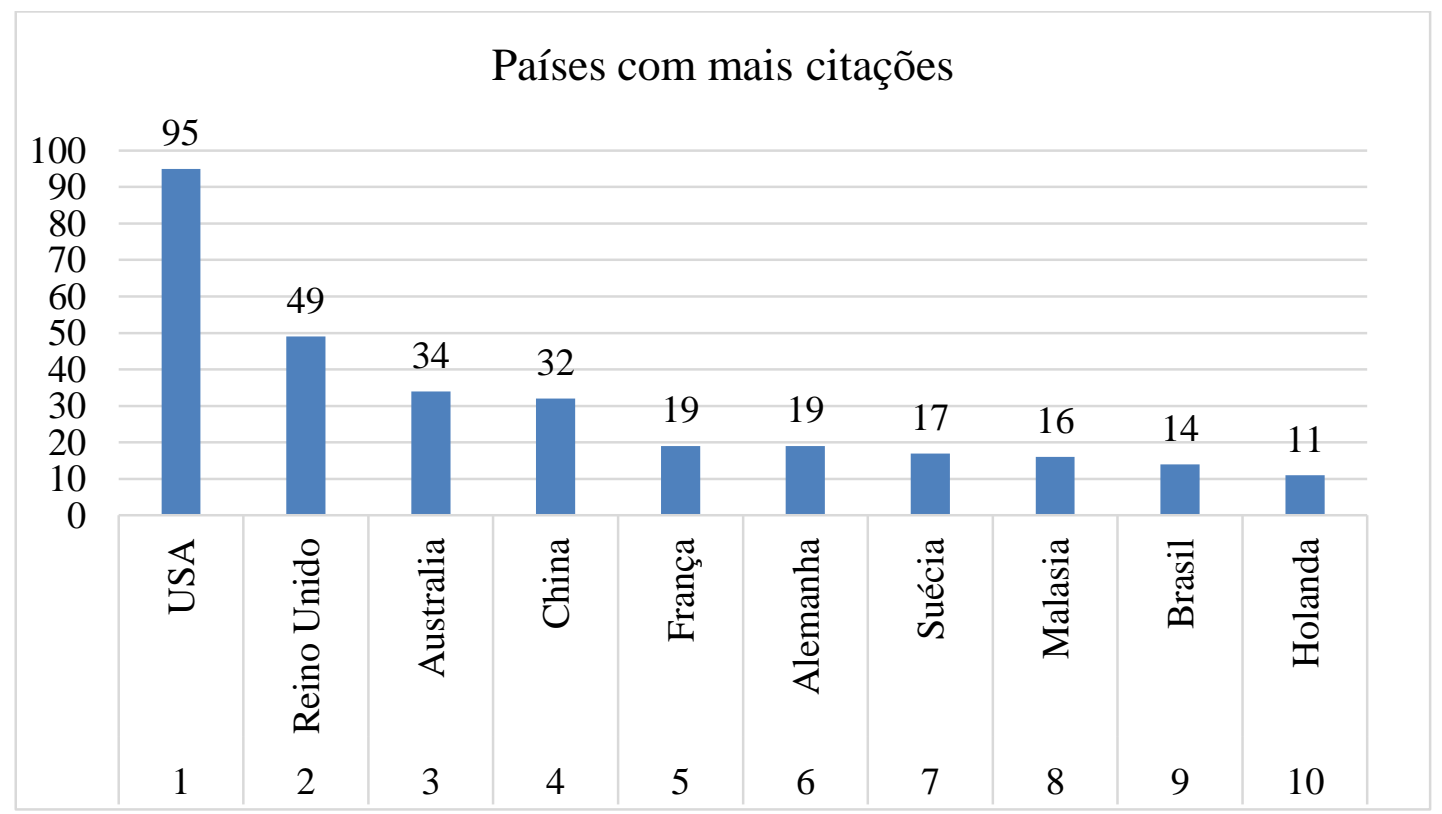

Figura 2 - Países com mais citações no TGCS

Fonte: elaborado pelos autores, a partir da Web of Science, julho de 2019.

Com base nas referências mais citadas, observam-se os trabalhos recentes e com mais citações nos últimos 5 anos, como exemplo a pesquisa de Joslin, R (Joslin, Robert); Muller, R (Muller, Ralf) publicado em 2015, intitulado Relationships between a project management methodology and project success in different project governance contexts, este artigo analisou a relação entre o uso de uma metodologia de gerenciamento de projetos (PMM) e o sucesso do projeto e o impacto do contexto de governança de projetos nessa relação. Os resultados do estudo mostram que a aplicação de um PMM conta para $22,3 \%$ da variação no sucesso do projeto, e os PMMs que são considerados suficientemente abrangentes para gerenciar o projeto levam a níveis mais elevados de sucesso do projeto do que os PMMs que precisam ser suplementado para uso pelo gerente de projeto.

Assim, apresentado a busca sistemática da pesquisa, a seguir passa-se a análise descritiva dos trabalhos selecionados, visando a continuidade do objetivo proposto para a presente pesquisa.

\subsection{Resultados da análise descritiva}

Conforme descrito na metodologia, a busca na base de dados Web of Science (WoS), de artigos internacionais que destacam a Teoria da Contingência e sua relação com as diversas abordagens, retornou em destaque os principais temas pesquisados, conforme se apresenta na tabela, abaixo.

Tabela 5 - Temas mais abordados

\begin{tabular}{lcc}
\hline \multicolumn{1}{c}{ Temas } & Artigos & \multicolumn{1}{l}{$\%$} \\
\hline Gestão & 153 & $47 \%$ \\
\hline Negócios & 91 & $28 \%$ \\
\hline Finanças empresarias & 32 & $10 \%$ \\
\hline Ciências Sociais e Gestão & 27 & $8,2 \%$ \\
\hline Engenharia Industrial & 26 & $7,9 \%$ \\
\hline Economia & 13 & $3,9 \%$ \\
\hline Gestão e Sustentabilidade & 11 & $3,3 \%$
\end{tabular}


O Estado da Arte sobre a Teoria da Contingência: um Estudo Bibliométrico entre o Período de 2015 a 2019

\begin{tabular}{lcc} 
Psicologia aplicada & 11 & $3,3 \%$ \\
\hline Sistema de Informação & 10 & $3,1 \%$ \\
\hline Gestão Ambiental & 10 & $3,1 \%$ \\
\hline Total & 326 & $3,1 \%$ \\
\hline
\end{tabular}

Fonte: elaborado pelos autores, a partir da Web of Science, julho de 2019.

Percebe-se de acordo com a Tabela 6, que a maioria dos artigos encontrados com a palavrachave "Contingency theory", as áreas do conhecimento como maior número de publicação nos períodos analisados, especificamente "Gestão" com $47 \%$ das publicações, seguidos de Negócio e Finanças com $28 \%$ e $10 \%$ respectivamente; os quais são possíveis de considerar a espinha dorsal dos debates sobre a Teoria da Contingência nos últimos cinco anos. Ademais, observase os 10 artigos indexados a um top 10 identificados na análise bibliométrica mais recente e contemporânea do ano de 2019.

Quadro 2: Trabalhos mais recentes

\begin{tabular}{|c|c|c|c|}
\hline Autores & Títulos dos trabalhos & $\begin{array}{l}\text { Fonte das } \\
\text { Publicações }\end{array}$ & Ano \\
\hline $\begin{array}{l}\text { Roman Pais Seles, Bruno } \\
\text { Michel; Lopes de Sousa } \\
\text { Jabbour, Ana Beatriz; } \\
\text { Chiappetta Jabbour, } \\
\text { Charbel Jose; et al. }\end{array}$ & $\begin{array}{l}\text { Do Environmental Practices Improve Business } \\
\text { Performance Even in an Economic Crisis? } \\
\text { Extending the Win-Win Perspective }\end{array}$ & $\begin{array}{l}\text { Ecological } \\
\text { Economics }\end{array}$ & 2019 \\
\hline $\begin{array}{l}\text { Hu, Xiaochen; Lovrich, } \\
\text { Nicholas P. }\end{array}$ & $\begin{array}{l}\text { Social media and the police A study of } \\
\text { organizational characteristics associated with } \\
\text { the use of social media }\end{array}$ & $\begin{array}{l}\text { Policing-An } \\
\text { International Journal of } \\
\text { Police Strategies \& } \\
\text { Management }\end{array}$ & 2019 \\
\hline $\begin{array}{l}\text { Turner, Rodney; Miterev, } \\
\text { Maxim }\end{array}$ & $\begin{array}{l}\text { The Organizational Design of the Project- } \\
\text { Based Organization }\end{array}$ & $\begin{array}{l}\text { Project Management } \\
\text { Journal }\end{array}$ & 2019 \\
\hline $\begin{array}{l}\text { Jiang, Lixin; } \quad \text { Lopez } \\
\text { Bohle, Sergio; Roche, } \\
\text { Maree }\end{array}$ & $\begin{array}{l}\text { Contingent Reward Transactional Leaders as } \\
\text { "Good Parents": Examining the Mediation } \\
\text { Role of Attachment Insecurity and the } \\
\text { Moderation Role of Meaningful Work }\end{array}$ & $\begin{array}{l}\text { Journal of Business } \\
\text { And Psychology }\end{array}$ & 2019 \\
\hline $\begin{array}{l}\text { Kahar, Suleman H. A.; } \\
\text { Ikbal, Muhammad; Jabid, } \\
\text { Abdullah W.; et al. }\end{array}$ & $\begin{array}{l}\text { Ethical Optimism, Participative Budgeting, } \\
\text { and Managerial Performance in Regional } \\
\text { Government Work Unit in Indonesia: A } \\
\text { Contingency Theory Approach }\end{array}$ & $\begin{array}{l}\text { Quality-Access } \\
\text { Success }\end{array}$ & 2019 \\
\hline $\begin{array}{l}\text { Goh, Shao Hung; } \\
\text { Eldridge, Stephen }\end{array}$ & $\begin{array}{l}\text { Sales and Operations Planning: The effect of } \\
\text { coordination mechanisms on supply chain } \\
\text { performance }\end{array}$ & $\begin{array}{l}\text { International Journal of } \\
\text { Production } \\
\text { Economics }\end{array}$ & 2019 \\
\hline $\begin{array}{l}\text { Baker, Marzena; Ali, } \\
\text { Muhammad; French, } \\
\text { Erica }\end{array}$ & $\begin{array}{l}\text { Effectiveness of gender equality initiatives in } \\
\text { project-based organizations in Australia }\end{array}$ & $\begin{array}{l}\text { Australian Journal of } \\
\text { Management }\end{array}$ & 2019 \\
\hline $\begin{array}{l}\text { Hutaibat, Khaled; } \\
\text { Alhatabat, Zaidoon }\end{array}$ & $\begin{array}{l}\text { Management accounting practices' adoption in } \\
\text { UK universities }\end{array}$ & $\begin{array}{l}\text { Journal of Further and } \\
\text { Higher Education }\end{array}$ & 2019 \\
\hline $\begin{array}{l}\text { Ortas, Eduardo; Burritt, } \\
\text { Roger Leonard; Christ, } \\
\text { Katherine Leanne }\end{array}$ & $\begin{array}{l}\text { The influence of macro factors on corporate } \\
\text { water management: A multi-country quantile } \\
\text { regression approach }\end{array}$ & $\begin{array}{l}\text { Journal of Cleaner } \\
\text { Production }\end{array}$ & 2019 \\
\hline $\begin{array}{l}\text { Fernandez-Robin, } \\
\text { Cristobal; Soledad } \\
\text { Celemin-Pedroche, }\end{array}$ & $\begin{array}{l}\text { Green Practices in Hospitality: A Contingency } \\
\text { Approach }\end{array}$ & Sustainability & 2019 \\
\hline
\end{tabular}


O Estado da Arte sobre a Teoria da Contingência: um Estudo Bibliométrico entre o Período de 2015 a 2019

\begin{tabular}{|l|l|l|l|}
\hline Maria; Santander- & & \\
Astorga, Paulina; et al. & & & \\
\hline
\end{tabular}

Fonte: elaborado pelos autores, a partir da Web of Science, julho de 2019.

O primeiro artigo analisado é intitulado "Do Environmental Practices Improve Business Performance Even in an Economic Crisis? Extending the Win-Win Perspective”, dos autores Roman Pais Seles, Bruno Michel; Lopes de Sousa Jabbour, Ana Beatriz; Chiappetta Jabbour, Charbel Jose; et al. Os pesquisadores, no estudo apresentaram, as práticas ambientais com grande influência no desempenho dos negócios mesmo em momentos de crise econômica? Voltado a perspectiva Lucros.

Como principais resultados, os autores apresentaram por meio de modelagem via PIS (PLS. PM), sendo os resultados discutidos através das lentes teóricas da teoria da contingência e da teoria das capacidades dinâmicas. Portanto, concluíram que as empresas podem beneficiar da consciência de que, mesmo em tempos difíceis, as práticas ambientais continuam a apoiar o desempenho dos negócios.

O Segundo trabalho no Top 10, é intitulado "Social media and the police A study of organizational characteristics associated with the use of social media", de autoria de $\mathrm{Hu}$, Xiaochen; Lovrich, Nicholas P. No artigo os autores pesquisaram sobre a finalidade da maioria das agências policiais nos EUA fazem a alegação de que eles usam a mídia social, e esse uso está desenhando uma grande atenção acadêmica. A maioria dos estudos sobre o uso policial de mídia social se concentrar no conteúdo da polícia sites de mídia social. Pouca pesquisa, entretanto, foi conduzida a respeito de que tipos de agências de polícia estão fazendo de fato o uso de meios sociais. $\mathrm{O}$ objetivo deste artigo é preencher essa lacuna no conhecimento a respeito das principais características organizacionais das agências policiais associadas ao uso de redes sociais Mídia.

O terceiro artigo publicado na revista Project Management Journal, dos autores Turner, Rodney; Miterev, Maxim. Este artigo investigou as opções de design organizacional e seus antecedentes para o design de organizações baseadas em projetos. A literatura existente reconhece a diversidade de formulários organizacionais baseados em projetos, mas nem as configurações específicas das dimensões de design organizacional nem as razões por trás de suas diferenças são amplamente abordadas. Com base em entrevistas de 12 organizações baseadas em projetos em vários setores, este estudo identificou três configurações comuns de projeto organizacional de organizações baseadas em projetos. Ao final concluiu a existência de uma escala de fatores internos e externos, que podem influenciar a escolha da configuração. Os achados são resumidos em cinco conclusões imperiosas.

Quanto ao quarto artigo de Jiang, Lixin; Lopez Bohle, Sergio; Roche, Maree, como título "Contingent Reward Transactional Leaders as "Good Parents": Examining the Mediation Role of Attachment Insecurity and the Moderation Role of Meaningful Work", os achados da pesquisa destacaram a analogia entre os líderes transacionais de recompensa contingente (caracterizado por clareza, consistência, consideração e conseqüências), desenvolvendo um modelo de mediação moderado onde a insegurança de apego media a relação da liderança transacional de recompensa contingente com a insegurança no trabalho e seu desempenho laboral e comportamebtal nas organizações.

Portanto, concluíram, os autores que o trablho apoiando a teoria de contingência possui significativa influencia com a relação entre a liderança transacional de recompensa contingente 
O Estado da Arte sobre a Teoria da Contingência: um Estudo Bibliométrico entre o Período de 2015 a 2019

e a prevenção e apego à ansiedade. Além disso, o efeito indireto da liderança transacional de recompensa contingente e dos resultados dos seguidor por meio de evitação e apego à ansiedade depende de um trabalho significativo.

Na sequência o quinto trabalho intitulado "Ethical Optimism, Participative Budgeting, and Managerial Performance in Regional Government Work Unit in Indonesia: A Contingency Theory Approach”, dos autores Kahar, Suleman H. A.; Ikbal, Muhammad; Jabid, Abdullah W.; et al.; os objetivo do estudo visou examinar empiricamente a influência da participação orçamentária na folga orçamentária e no desempenho gerencial do governo da província de Maluku - Indonésia. As implicações teóricas desta pesquisa são que a teoria da Agência e a teoria da contingência podem ser utilizadas como complemento de um processo de participação orçamentário mais efetivo, integrando a mensuração do desempenho.

Em contraponto, as mais recentes pesquisas sobre a Teoria da Contingência, destaca-se o sexto trabalho, intitulado "Sales and Operations Planning: The effect of coordination mechanisms on supply chain performance", dos autoresGoh, Shao Hung; Eldridge, Stephen. Os pesquisadores investigaram o efeito das organizações sobre o desempenho da cadeia de suprimentos, utilizando a perspectiva das teorias de coordenação e contingência.

O sétimo trabalho, denominado "Effectiveness of gender equality initiatives in project-based organizations in Australia", dos autores Baker, Marzena; Ali, Muhammad; French, Erica. Os resutlados da pesquisa destacaram que pouco se sabe sobre o impacto das iniciativas de igualdade de gênero no trabalho e a participação das mulheres. Avaliaram a sua eficácia nos níveis de representação das mulheres em organizações de propriedade e mineração australianas. Os resultados descaram oa amostra de 358 organizações com um atraso de tempo de 2 anos nas promoções e participação das mulheres na empresas pesquisadas.

O oitavo trabalho de Hutaibat, Khaled; Alhatabat, Zaidoon destacou "Management accounting practices' adoption in UK universities". O artigo apresentou o seguinte objetivo: explorar a extensão e os determinantes da adoção das práticas contábeis gerenciais nas universidades do Reino Unido. Nesta veia, a análise de regressão conclui que apenas sete das vinte e nove variáveis iniciais são orientadas para a estratégica, a cultura inovadora, a trialabilidade, os fatores institucionais miméticos e normativos e o tipo de Universidade são determinados como os preditores de mapas aprimorados nas universidades do Reino Unido. Os achados do presente estudo são úteis para consideração ao se olhar para a adoção de novas práticas contábeis gerenciais em instituições de ensino superior.

O penúltimo trabalho no Top 10 no ano de 2019, tem o título "The influence of macro factors on corporate water management: A multi-country quantile regression approach", dos autores: Ortas, Eduardo; Burritt, Roger Leonard; Christ, Katherine Leanne. O artigo baseado na teoria de contingência examinou a extensão em que os fatores macro puderam afetar práticas corporativas da gerência de água de uma amostra de 3729 companhias alistadas que operam-se em 59 países diferentes. Os resultados da modelagem de regressão Quantil revelam taxas de crescimento populacional dos paises, eventos climáticos extremos e aridez estão ligados às práticas corporativas de gestão de água dos líderes. Dadas as descobertas, sugestões para melhorar a gestão da água corporativa são feitas para os decisores políticos governamentais e gestores de empresas com diferentes qualidade das práticas de gestão da água.

O décimo artigo analisado, é intitulado por "Green Practices in Hospitality: A Contingency Approach", desenvolvido pelos autores Fernandez-Robin, Cristobal; Soledad Celemin- 
O Estado da Arte sobre a Teoria da Contingência: um Estudo Bibliométrico entre o Período de 2015 a 2019

Pedroche, Maria; Santander-Astorga, Paulina; et al. O artigo centrou-se na descoberta de práticas sustentáveis e possíveis práticas inovadoras de acordo com fatores da teoria das contingências. Para tanto, foi realizada uma abordagem qualitativa. O estudo analisou 24 hotéis independentes. Foram utilizadas entrevistas semiestruturadas e conduzidas pessoalmente com os gestores de cada hotel. Os resultados mostram que, de acordo com a teoria de contingência, o comportamento organizacional é determinado pelo ambiente em que o Hotel Opera, o tamanho do estabelecimento, onde os hotéis de grande e médio porte estão mais comprometidos com a sustentabilidade desenvolvimento, a tecnologia ambiental adotada e implementada pelo Hotel, e o principal tipo de clientela, com Hotéis voltados para viajantes de negócios que mostrem maior atenção ao meio ambiente do que aqueles voltados para os viajantes de lazer, principalmente associados com valores sócio-culturais.

Os autores verificaram que os fatores que não foram completamente decisivos nos resultados analisados foram a idade do hotel e o sexo do dono do hotel. Considerando os resultados alcançados, este estudo também podem contribuir para a identificação dos hotéis mais sustentáveis e pode ajudar as empresas Hoteleiras a entenderem e colherem os benefícios de seguir um caminho sustentável.

\section{CONSIDERAÇÕES FINAIS}

O objetivo do presente trabalho foi analisar como se apresenta a produção cientifica internacional sobre a Teoria da Contingência na Web of Science (WoS) entre os anos de 2015 e 2019, através dos artigos publicados com maior grau de impacto, países mais produtivos, os autores mais citados, as referências mais utilizadas entre outros, por meio de uma análise bibliométrica. A partir da análise bibliométrica dos artigos indexados na base de dados Web of Science (WoS), foram localizados 326 artigos, sendo escritos por 840 pesquisadores, com vínculo em 452 instituições de 64 países, publicados em 210 periódicos e, utilizaram 19.113 referências, bem como 1.072 palavras-chave. Demonstrando o crescente interesse dos pesquisadores pela temática Teoria da Contingência. Foram também, analisados os 10 mais recentes, e estes podem ser tratados como relevantes insumos para pesquisadores e estudantes universitários e outros interessados na temática.

Como resultado observou-se também, que a pesquisa sobre a Teoria da Contingência está em ascensão nos últimos anos e os pesquisadores como maior número de citações nos últimos cinco anos o trabalho dos americanos Josefy M, Kuban S, Ireland RD, Hitt MAde 2015 com maior número de citações (60 citações), publicado na revista Academy Of Management Annals, intitulado, "All ThingsGreat and Small: OrganizationalSize, Boundaries of the Firm, and a ChangingEnvironment".

Adicionalmente foram mapeados os periódicos mais relevantes da área. Para isso foram considerados dois indicadores: (a) quantidade de artigos publicados, e (b) grau de impacto, medido pelo número de citações no WoS. Isso gerou uma lista com os periódicos que mais publicaram sobre o tema, sendo destaque a revista Journal of Cleaner Productioncom o que mais publicou artigos no tema Teoria da Contingência (7 artigos), constando como fontes de publicações com maior impacto, tendo em vista que apresenta o score de 163 citações.

Chama atenção também, os países que mais publicam sobre o tema os Estados Unidos aparecem com 95 publicações, que correspondem aproximadamente $29 \%$ dos artigos encontrados na pesquisa, e o Brasil em que figurou na $9^{\circ}$ posição entre os 10 países que mais publicaram sobre o tema. E dentre os artigos mais recentes, destaca-se o artigo intitulado "Green Practices in 
O Estado da Arte sobre a Teoria da Contingência: um Estudo Bibliométrico entre o Período de 2015 a 2019

Hospitality: A Contingency Approach", desenvolvido pelos autores Fernandez-Robin, Cristobal; Soledad Celemin-Pedroche, Maria; Santander-Astorga, Paulina; et al. Com isso, buscou apresentar um panonoram das publicações, quais sejam, a base de dados selecionada, o corte temporal, os temas, as citações, e outros.

E ainda, respondendo ao problema de pesquisa, os achados da literatura são os mais diversos possíveis, como sugestão recomenda-se para futuras pesquisas, ampliar o número de artigos analisados, incluindo um comparativo com a produção científica internacional e nacional, através da bibliometria e sociometria sob o enfoque de small worlds e graus de centralidade.

\section{REFERÊNCIAS}

Aguiar, A. B. D., \& Frezatti, F. (2007). Sistema de controle gerencial e contextos de processo de estratégia: contribuições da teoria da contingência. Anais.

Albano Soares, Y. M., Nóbrega Cavalcante, P. R., \& Dantas dos Santos, Y. (2019). Informações contingências: um estudo sobre a percepção de gestores do setor de óleo e gás no Brasil. Capital Científico, 17(3).

Baker, M., Ali, M., \& French, E. (2018). Effectiveness of gender equality initiatives in projectbased organizations in Australia. Australian Journal of Management, 0312896218805809.

Beuren, I. M., \& Fiorentin, M. (2014). Influência de Fatores Contingenciais nos Atributos do Sistema de Contabilidade Gerencial: um estudo em empresas têxteis do Estado do Rio Grande do Sul. Revista de Ciências da Administração, 16(38), 195-212.

Burns, T., \& Stalker, G. M. (1961). The Management of Innovation (London: Tavistock, 1961). BurnsThe Management of Innovation 1961.

Čadež, S. (2007). A configuration form of fit in management accounting contingency theory: an empirical investigation.

Camacho, R. R. (2010). Fatores condicionantes da gestão de custos interorganizacionais na cadeia de valor de hospitais privados no Brasil: uma abordagem à luz da Teoria da Contingência (Doctoral dissertation, Universidade de São Paulo).

Chandler, A. D. (1990). Strategy and structure: Chapters in the history of the industrial enterprise (Vol. 120). MIT press.

Chen, S. (2008). DCF techniques and nonfinancial measures in capital budgeting: a contingency approach analysis. Behavioral Research in Accounting, 20(1), 13-29.

Chenhall, R. H. (2003). Management control systems design within its organizational context: findings from contingency-based research and directions for the future. Accounting, organizations and society, 28(2-3), 127-168.

Donaldson, L. (1999). Teoria da contingência estrutural. Handbook de estudos organizacionais, 1, 105-133.

Drazin, R., \& Van de Ven, A. H. (1985). Alternative forms of fit in contingency theory. Administrative science quarterly, 514-539.

Espejo, M. M. D. S. B, \& Frezatti, F. (2008). Perfil dos atributos do sistema orçamentário sob a perspectiva contingencial: uma abordagem multivariada (Doctoral dissertation, Universidade de São Paulo).

Fernández-Robin, C., Celemín-Pedroche, M. S., Santander-Astorga, P., \& Alonso-Almeida, M. D. M. (2019). Green Practices in Hospitality: A Contingency Approach. Sustainability, 11(13), 3737.

Fagundes, J. A., Soler, C. C., Lavarda, C. E. F., \& Lavarda, R. (2011). Gestão do curso de administração considerando o enfoque da teoria da contingência. Revista de Contabilidade do Mestrado em Ciências Contábeis da UERJ, 14(3), 44-59. 
O Estado da Arte sobre a Teoria da Contingência: um Estudo Bibliométrico entre o Período de 2015 a 2019

Fagundes, J. A., Petri, M., Lavarda, R. B., Rodrigues, M. R., Lavarda, C. E. F., \& Soller, C. C. (2011). Estrutura organizacional e gestão sob a ótica da teoria da contingência. Gestão \& Regionalidade, 26(78).

Goh, S. H., \& Eldridge, S. (2019). Sales and Operations Planning: The effect of coordination mechanisms on supply chain performance. International Journal of Production Economics, 214, 80-94.

Henri, J. F. (2006). Management control systems and strategy: A resource-based perspective. Accounting, organizations and society, 31(6), 529-558.

Hutaibat, K., \& Alhatabat, Z. (2019). Management accounting practices' adoption in UK universities. Journal of Further and Higher Education, 1-15.

Jiang, L., Bohle, S. L., \& Roche, M. (2018). Contingent Reward Transactional Leaders as "Good Parents": Examining the Mediation Role of Attachment Insecurity and the Moderation Role of Meaningful Work. Journal of Business and Psychology, 1-19.

Junqueira, E. R. (2010). Perfil do sistema de controle gerencial sob a perspectiva da teoria da contingência (Doctoral dissertation, Tese de Doutorado em Ciências Contábeis, Universidade de São Paulo, São Paulo, Brasil).

Josefy, M., Kuban, S., Ireland, R. D., \& Hitt, M. A. (2015). All things great and small: Organizational size, boundaries of the firm, and a changing environment. The Academy of Management Annals, 9(1), 715-802.

Hu, X., \& Lovrich, N. P. (2019). Social media and the police: A study of organizational characteristics associated with the use of social media. Policing: An International Journal.

Kahar, S. H., Ikbal, M., Jabid, A. W., \& Purbaya, A. (2019). Ethical Optimism, Participative Budgeting, and Managerial Performance in Regional Government Work Unit in Indonesia: A Contingency Theory Approach. Calitatea, 20(171), 70-75.

Klein, S. B., Trocz, P. J. O., Toigo, L. A., \& Wrubel, F. (2019). Relações entre tipos de sistemas orçamentários e fatores contingenciais no setor industrial. In Anais XIII Congresso Anpcont-SP.São Paulo.

Lorsch, J. W., \& Lawrence, P. R. (1967). Organization and environment: Managing differentiation and integration. Boston: Division of Research, Graduate School of Business Administration, Harvard University.

das Neves Machado, R. (2007). Análise cientométrica dos estudos bibliométricos publicados em periódicos da área de biblioteconomia e ciência da informação (19902005). Perspectivas em ciência da informação, 12(3), 2-20.

Mainardes, e., Silveira, a., Gomes, g., \& Deschamps, m. (2008). Produção científica brasileira em marketing de serviços: análise dos eventos EnANPAD e EMA, 2003-2006. Revista Base (Administração e Contabilidade) da UNISINOS, 5(2), 142-153.

Marques, K. C. M., \& Souza, R. P. (2010). Pontos críticos da abordagem da contingência nos estudos da contabilidade gerencial. In Anais do Congresso Brasileiro de Custos-ABC.

Oliveira, D. P. R. (2008). Teoria geral da administração. São Paulo: Atlas.

Oliveira, J. C. (2001). Estudo bibliométrico das publicações de custos em enfermagem no período de 1966 a 2000 [dissertação]. São Paulo: Escola de Enfermagem, Universidade de São Paulo.

Ortas, E., Burritt, R. L., \& Christ, K. L. (2019). The influence of macro factors on corporate water management: A multi-country quantile regression approach. Journal of Cleaner Production, 226, 1013-1021.

Otley, D. (2016). The contingency theory of management accounting and control: 1980-2014. Management accounting research, 31, 45-62.

Seles, B. M. R. P., de Sousa Jabbour, A. B. L., Jabbour, C. J. C., Latan, H., \& Roubaud, D. (2019). Do Environmental Practices Improve Business Performance Even in an Economic Crisis? Extending the Win-Win Perspective. Ecological Economics, 163, 189-204. 
O Estado da Arte sobre a Teoria da Contingência: um Estudo Bibliométrico entre o Período de 2015 a 2019

Stoner, J. A., \& Freeman, R. E. (1985). Administração. $5^{\text {a }}$ edição. Rio de Janeiro: Printice-Hall do Brasil.

Tillema, S. (2005). Towards an integrated contingency framework for MAS sophistication: Case studies on the scope of accounting instruments in Dutch power and gas companies. Management Accounting Research, 16(1), 101-129.

Turner, R., \& Miterev, M. (2019). The Organizational Design of the Project-Based Organization. Project Management Journal, 8756972819859746.

Vanti, N. A. P. (2002). Da bibliometria à webometria: uma exploração conceitual dos mecanismos utilizados para medir o registro da informação e a difusão do conhecimento. Ciência da informação, 31(2), 152-162.

Woodward, J. (1958). Management and technology (No. 3). HM Stationery Off. 